\title{
Extending the Range of Blood Glucose Measurements with Dextrostix and a Reflectance Meter
}

\author{
I. N. Scobie, H. Y.Son, B. H. Tey and P.H. Sönksen \\ Department of Medicine, St Thomas's Hospital Medical School, London, UK
}

Summary. The useful range of blood glucose measurements with Dextrostix can be extended by varying the incubation time and making appropriate corrections to the observed values.
Key words: Dextrostix, reflectance meter, blood glucose.
Dextrostix reagent strips use a glucose oxidase enzymatic reaction which is incomplete after $3 \mathrm{~min}$. The measured blood glucose concentration rises progressively the longer the blood is allowed to remain in contact with the reagent pad. The aim of this study was to determine how this aspect of Dextrostix could extend the range of these test strips.

\section{Methods}

Thirty blood samples were collected from non-diabetic patients, diabetic patients and patients undergoing an insulin tolerance test to obtain a wide range of glucose concentrations. Samples were collected into lithium heparin tubes and maintained at $34-37{ }^{\circ} \mathrm{C}$ in a water bath. Drops of blood from each sample were placed on the Dextrostix reagent pad and left in contact for exactly $15,30,45,60,90,120$ and

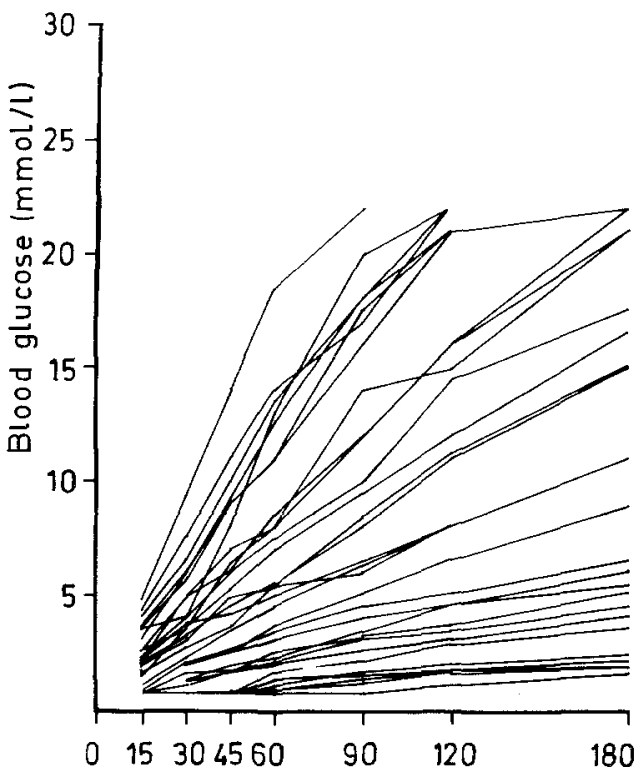

a

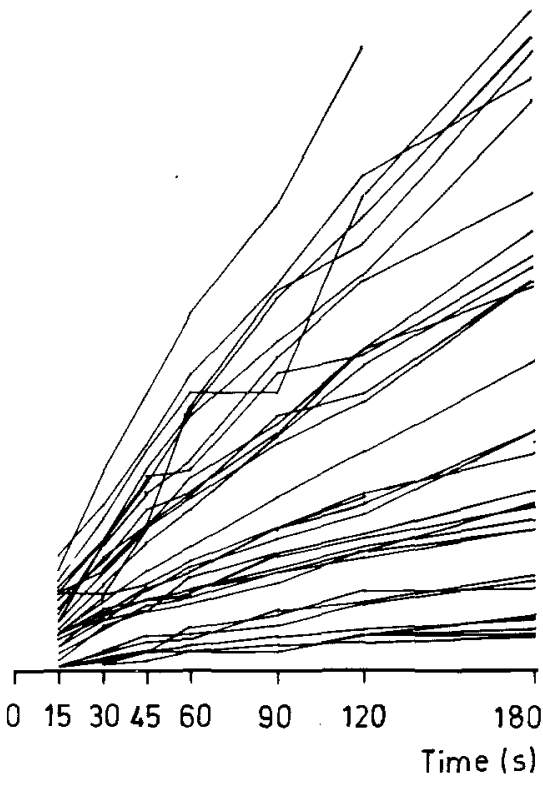

Fig. $1 \mathrm{a}$ and $b$. Blood glucose readings obtained on Eyetone (a) and Glucochek (b) meters after various time intervals of incubation of blood with the Dextrostix 


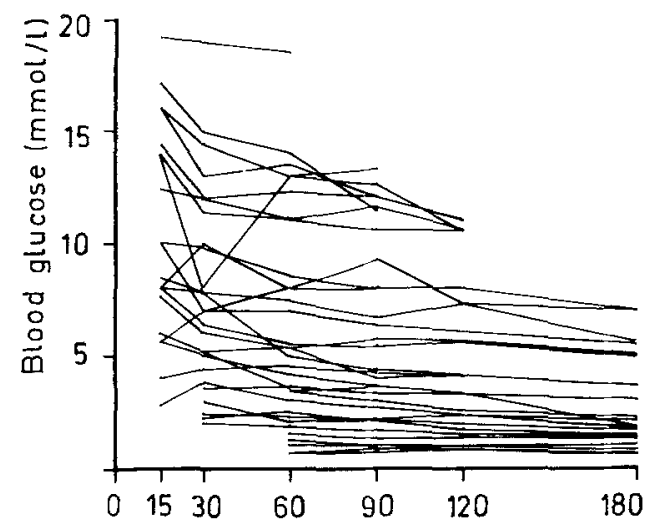

a

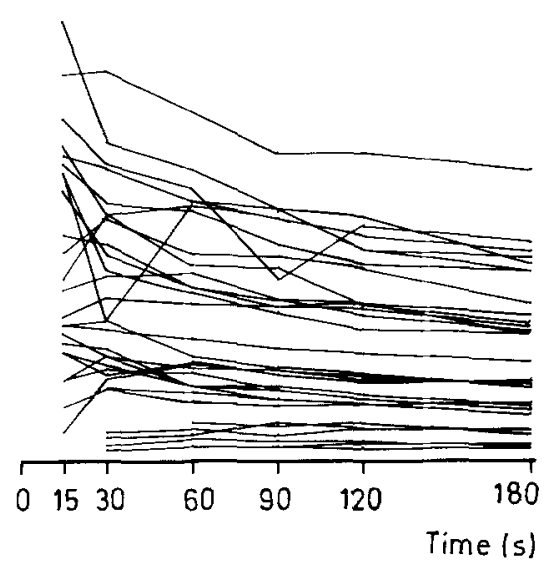

$b$
Fig. 2 a and b. A comparison of blood glucose measurements obtained on Eyetone (a) and Glucochek (b) meters after incubation periods ranging from 15 to $180 \mathrm{~s}$
180 s. In addition, a further ten samples were left in contact with the reagent at 30,35,60 and $65 \mathrm{~s}$ to determine the effect of a small timing error $(5 \mathrm{~s})$. The strips were then read on an Eyetone (Ames Division, Miles Laboratories, Slough, Bucks, UK) and a Glucochek meter (Medistron, Crawley, Sussex, UK). Two additional samples were incubated with glucose to produce very high concentrations (33 and $39 \mathrm{mmol} / \mathrm{l}$, respectively).

\section{Results}

Blood glucose readings on the Eyetone and Glucochek meters after the various time intervals are shown in Figure 1 (range at $60 \mathrm{~s} 0.6-18.5 \mathrm{mmol} / 1$ ). Increasing the contact time of the blood on the reagent strip produced a steadily progressive rise in the measured blood glucose concentration as would be expected from the nature of the glucose-oxidase reaction. There was a slight difference between the values obtained using the Eyetone and Glucochek meters but this was not statistically significant.

In Figure 2, for each blood sample the meter reading at $15 \mathrm{~s} \times 4,30 \mathrm{~s} \times 2,60 \mathrm{~s}, 90 \mathrm{~s} \times 0.67,120 \mathrm{~s} \times 0.5$ and $180 \mathrm{~s} \times 0.33$ is plotted and then each point for a given blood sample is joined by a straight line. If the technique is to give accurate results then for each sample a straight horizontal line should result which passes through the 'true' $60 \mathrm{~s}$ value. This was generally the case except for the $15 \mathrm{~s} \times 4$ value.

The Eyetone meter is calibrated to read as low as $0.6 \mathrm{mmol}$ but its accuracy at this level is often questioned. We found that leaving blood in contact with the reagent pad for 120 or $180 \mathrm{~s}$ and dividing the value obtained by 2 or 3 respectively gave a confirmatory (and probably more accurate) measure of the degree of hypoglycaemia. When the blood glucose was very high ( $>22 \mathrm{mmol} / \mathrm{l}$ ), a reading could not be obtained on the Eyetone without shortening the incubation time (e.g. 30 s value $\times 2$ ) when a more precise measurement was possible. Two blood samples with glucose concentrations of 33 and $39 \mathrm{mmol} / 1$ were analysed using this technique. The $30 \mathrm{~s}$ value $\times 2$ for the $33 \mathrm{mmol} / 1$ blood was $34 \mathrm{mmol} / 1$ (Eyetone) and $34.4 \mathrm{mmol} / \mathrm{l}$ (Glucochek) and for the $39 \mathrm{mmol} / 1$ sample the results were $36 \mathrm{mmol} / \mathrm{l}$ (Eyetone) and $37 \mathrm{mmol} / 1$ (Glucochek). In a separate set of experiments we found that with incubation times of 30 and $60 \mathrm{~s}$ the effect of a small timing error $(5 \mathrm{~s})$ was so small as to be of no clinical significance.

\section{Discussion}

Our results indicate that it is not always necessary to read Dextrostix after a contact time of $60 \mathrm{~s}$ and we have shown that by altering this incubation time the useful range of Dextrostix may be extended. This may not be relevant in the context of home blood glucose monitoring by diabetic patients, but clearly is for doctors treating hyperglycaemic or hypoglycaemic emergencies.

Received: 4 October 1982

and in revised form: 10 May 1983

Dr. I. N. Scobie

Department of Medicine

St. Thomas's Hospital Medical School

London SE1 7EH, UK 